\title{
CHRONIC NONPULSATILE BLOOD FLOW. III. EFFECTS OF PUMP FLOW RATE ON OXYGEN TRANSPORT AND UTILIZATION IN CHRONIC NONPULSATILE BIVENTRICULAR BYPASS
}

Ryuji Tominaga, MD

William Smith, DEng

Alex Massiello, ME

Hiroaki Harasaki, MD, PhD

Leonard A. R. Golding, MD
The relationship between blood flow and oxygen transport was studied in five calves with chronic nonpulsatile biventricular bypass. Seven days was allowed for recovery from the effects of anesthesia and operation; the natural heart was then fibrillated. Pump flows were maintained at nominal rates of 90,100 , or $120 \mathrm{ml} \cdot \mathrm{kg}^{-1} \cdot \min$ for 1 week each, with the sequence varied from experiment to experiment. Venous and arterial blood samples were taken at rest for blood gas analysis. Serum lactate analysis was done twice a week, on the third and seventh days after each pump flow change. Serum catecholamine levels were assayed on the seventh day of each flow rate. Progressive exercise tests were also conducted during each test segment. Basal oxygen consumption of a 4 -month-old calf was $6.3 \pm$ $0.3 \mathrm{ml} \cdot \mathrm{kg}^{-1} \cdot \mathrm{min}^{-1}$. The mixed venous oxygen tension decreased when pump flow rate was reduced $(29.6 \pm 1.0,28.3 \pm 1.2$, and $23.8 \pm 0.9 \mathrm{~mm} \mathrm{Hg}$ at 120,100 , and $90 \mathrm{ml} \cdot \mathrm{kg}^{-1} \cdot \mathrm{min}^{-1}$ of pump flow, respectively), and oxygen extraction increased linearly when pump flow rate was reduced. Hemoglobin concentration significantly affected oxygen extraction rate. Serum lactate concentration increased significantly at a $90 \mathrm{ml} \cdot \mathrm{kg}^{-1} \cdot \mathrm{min}^{-1}$ perfusion compared with concentrations at other pump flow rates $(7.81 \pm$ $2.42 \mathrm{mEq} / \mathrm{L}$ at $90 \mathrm{ml} \cdot \mathrm{kg}^{-1} \cdot \mathrm{min}^{-1}$ vs $0.71 \pm 0.19$ and $0.73 \pm 0.81 \mathrm{mEq} / \mathrm{L}$ at 100 and $120 \mathrm{ml} \cdot \mathrm{kg}^{-1} \cdot \mathrm{min}^{-1}$, respectively; $p<0.01$, analysis of variance, Scheffe $\boldsymbol{F}$ test). Maximum oxygen extraction during exercise was $78 \%$. These results suggest that a critical flow level between 90 and 100 $\mathrm{ml} \cdot \mathrm{kg}^{-1} \cdot \mathrm{min}^{-1}$ maintains oxidative metabolism in the calf with chronic nonpulsatile flow. The resulting oxygen delivery was slightly higher than that indicated in the literature. Maximal oxygen extraction was normal. (J Thorac Cardiovasc Surg 1996;111:863-72)
$\mathrm{T}^{\mathrm{t}}$ he acceptability and pathophysiology of nonpulsatile blood flow have been the subject of continuing controversy. ${ }^{1-3}$ Despite these debates, continuous-flow blood pumps have grown in popularity, not only for surgical cardiopulmonary bypass but

From the Department of Biomedical Engineering, Research Institute, The Cleveland Clinic Foundation, Cleveland, Ohio.

Supported by a research grant from the G. Herold and Leila Y. Mathers Charitable Foundation.

Received for publication Feb. 21, 1995; accepted for publication June 7, 1995.

Address for reprints: Ryuji Tominaga, MD, Division of Cardiovascular Surgery, Research Institute of Angiocardiology, Faculty of Medicine, Kyushu University, 3-1-1 Maedashi, Higashi-ku, Fukuoka, 812, Japan.

Copyright (C) 1996 by Mosby-Year Book, Inc.

$0022-5223 / 96 \$ 5.00+0 \quad \mathbf{1 2} / \mathbf{1} / \mathbf{6 6 8 2 1}$ also for short-term ventricular support and as a bridge to transplantation. ${ }^{4-6}$ In research applications, animals have been maintained for as long as 99 days with chronic nonpulsatile blood flow. ${ }^{7-9}$ The major research issues now center on how mammalian physiology compensates for the change in flow regimen.

One basic issue that has been studied in cardiorespiratory physiology is the concept of critical oxygen delivery. ${ }^{10}$ There are no reports, however, on the critical flow level for maintaining oxidative metabolism in chronic nonpulsatile blood flow. Such results could be compared with pulsatile flow studies to better understand the mechanics of nonpulsatile survival. Maximal oxygen extraction during exercise would further indicate the mammalian system's ability to adapt to and compensate for the continuousflow regimen. 


\section{Material and methods}

All animals in this study were managed in compliance with the "Principles of Laboratory Animal Care" formulated by the National Society for Medical Research and the "Guide to the Care and Use of Laboratory Animals" prepared by the Institute of Laboratory Animal Resources and published by the National Academy of Sciences (NIH Publication No. 8023, revised 1978).

Details of the experimental preparation are as described in the first article of this series. ${ }^{11}$ The implants were performed in 4-month-old calves weighing 89.8 to $93.6 \mathrm{~kg}$. The natural heart was bypassed, right ventricle to pulmonary artery and left ventricle to aorta, with externally mounted Hemadyne centrifugal pumps manufactured by the now defunct Hemadyne division of Medtronic, Inc. (Minneapolis, Minn.). On the seventh postoperative day, the natural heart was fibrillated and one of three pump flow levels was set.

A total of 30 exercise studies were performed on five animals with an animal treadmill (Animil; Gullwing, Inc., Sandusky, Ohio). The speed of the treadmill was increased from 0.3 to $2.1 \mathrm{mph}$ in increments of $0.2 \mathrm{mph}$, with each speed being maintained for 3 minutes. Treadmill slope was zero. Hemodynamic values were monitored continuously and recorded. Pulmonary artery and systemic arterial blood samples were drawn at the end of each speed setting and a blood gas analysis was performed with a Co-Oximeter 282 and an IL System 1302 (Instrumentation Laboratory, Co., Lexington, Mass.). The exercise study was performed twice a week, on the third and seventh days after establishment of each flow rate. There were no statistically significant differences with respect to when the postoperative exercise studies were performed for each flow rate. The body weight of the calf was measured immediately after exercise, and the body temperature was measured before exercise. Exercise studies were performed at mean times after operation of $18.5 \pm$ $1.9,15.3 \pm 1.8$, and $14.8 \pm 1.4$ days at pump flow rates of 90,100 , and $120 \mathrm{ml} \cdot \mathrm{kg}^{-1} \cdot \mathrm{min}^{-1}$, respectively. Pump flows were monitored by transit-time ultrasonic flow probes clamped on pump outflow cannulas (Transonic Systems, Inc., Ithaca, N.Y.). Exercise was terminated when the animal refused to continue or achieved a treadmill speed of $2.1 \mathrm{mph}$.

All data were divided into three groups according to pump flow rate. To investigate the effect of hemoglobin level on oxygen transport, the data were also divided into three groups according to hemoglobin concentration. Serum lactate measurements were obtained twice a week, on the third and seventh days after establishment of each pump flow setting. Serum catecholamine levels, including norepinephrine, epinephrine, and dopamine, were measured on the seventh day of each nominal flow rate. Blood samples were drawn only when the animal was calm, afebrile, and not cudding. All animals showed a mild to moderate degree of anemia because blood transfusion was restricted to the operative day or the first postoperative day to minimize changes in hematologic values. Centrifugal pump heads had to be changed once or twice during a 4-week experiment as a result of shaft seal failure or protein formation in the pump head. All data, including hemodynamic data, blood chemistry analyses, and blood gas analyses, were excluded if collected soon after pump head changes. The calves were electively killed after 4 weeks and underwent a full autopsy.

Previous studies in an earlier series of animals ${ }^{7-9}$ discussed left-right flow differences with mechanical blood pumps, and our previous practice during nonpulsatile biventricular bypass studies was to use the right side, lower flow as the effective cardiac output. During these experiments, however, one set of side holes on the right side inflow cannula sometimes migrated to the arterial side of the pulmonary valve, producing a variable rightside regurgitation. We therefore used left pump flows for calculation of the results reported here, recognizing that they may be $10 \%$ to $20 \%$ higher than the right-side values used earlier.

Catecholamine levels were measured with high-performance liquid chromatography. Including the small amount of dissolved oxygen, $\mathrm{VO}_{2}$ was calculated from the following formula:

$$
\begin{array}{r}
\dot{\mathrm{VO}}_{2}=\text { Cardiac output } \times\left[\mathrm{SaO}_{2}-\mathrm{SvO}_{2} \times \mathrm{Hb} \times 1.36+\right. \\
\left.0.003 \times\left(\mathrm{PaO}_{2}-\mathrm{PvO}_{2}\right)\right]
\end{array}
$$

where $\mathrm{VO}_{2}$ is oxygen consumption, $\mathrm{SaO}_{2}$ is arterial oxygen saturation, $\mathrm{SvO}_{2}$ is mixed venous oxygen saturation, $\mathrm{Hb}$ is hemoglobin concentration, $\mathrm{PaO}_{2}$ is arterial oxygen tension, and $\mathrm{PvO}_{2}$ is mixed venous oxygen tension. Oxygen extraction was determined from the following formula:

$$
\mathrm{O}_{2} \text { extraction }=\left(\mathrm{CaO}_{2}-\mathrm{CvO}_{2}\right) / \mathrm{CaO}_{2}
$$

where $\mathrm{CaO}_{2}$ is arterial oxygen content and $\mathrm{CvO}_{2}$ is venous oxygen content.

Data are reported as mean \pm standard error of the mean. Data were stored in and analyzed with a personal computer. Statistical significance was evaluated with the two-tailed, unpaired $t$-test. Analysis of variance and Scheffe $F$-test were used to evaluate the significance among three or four groups of data. Significance was assigned at $p<0.05$.

\section{Results}

At rest. Resting $\dot{\mathrm{V}}_{2}$ is shown on Table I. The oxygen extractions at 100 and $120 \mathrm{ml} \cdot \mathrm{kg}^{-1} \cdot \mathrm{min}^{-1}$ were significantly different from that at 90 $\mathrm{ml} \cdot \mathrm{kg}^{-1} \cdot \mathrm{min}^{-1}$, and the resting lactate level was significantly higher at $90 \mathrm{ml} \cdot \mathrm{kg}^{-1} \cdot \mathrm{min}^{-1}$. Also at $90 \mathrm{ml} \cdot \mathrm{kg}^{-1} \cdot \mathrm{min}^{-1}$ of pump flow, serum lactate concentration measured on the seventh day after pump flow establishment was higher than that on the third day $(9.78 \pm 3.76$ vs $6.14 \pm 3.3 \mathrm{mEq} / \mathrm{L})$, but this was not a statistically significant difference. Blood pressure, hemoglobin concentration, oxygen saturation, and $\dot{\mathrm{VO}}_{2}$ showed no significant differences among the three flow settings. Animal position (sitting or standing), hemoglobin concentration, and body temperatures were not found to have an effect on basal $\mathrm{VO}_{2}$. Mixed venous oxygen saturation and oxygen extraction correlated linearly with pump 


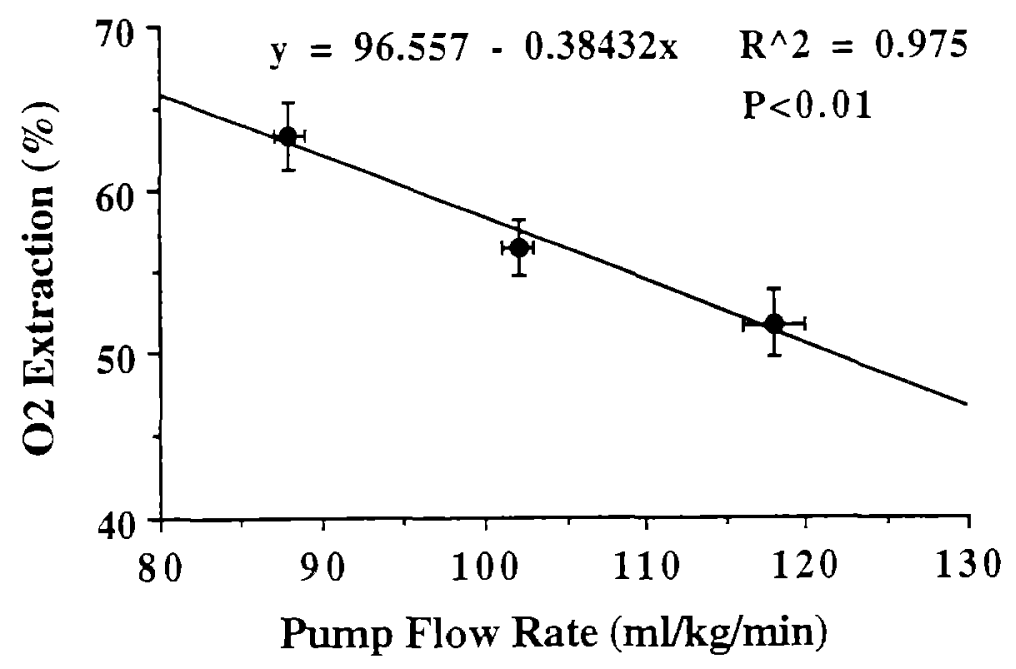

Fig. 1. Correlation between oxygen extraction $\left(\mathrm{O}_{2}\right.$ Extraction) and the left pump flow rate. Oxygen extraction correlated significantly with left pump flow. Small bars represent standard error of the mean.

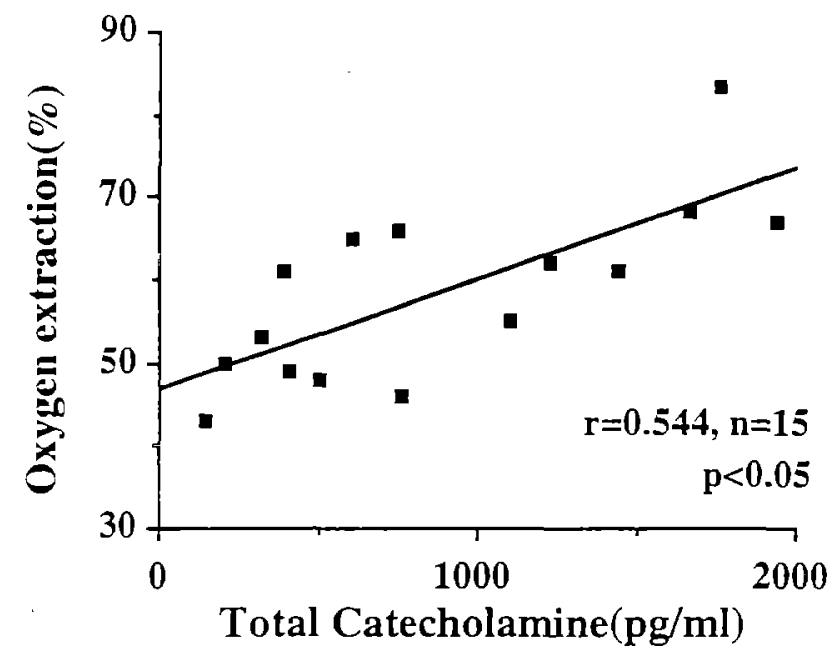

Fig. 2. Correlation between oxygen extraction and total catecholamine level. A significant $(p<0.05)$ correlation was observed.

flow rate $(p<0.01$; Fig. 1). During the first week of the experiment, before the natural heart was fibrillated, the mixed venous oxygen saturation was $62.3 \% \pm 2.2 \%$, which was significantly higher than the values at 90,100 , and $120 \mathrm{ml} \cdot \mathrm{kg}^{-1} \cdot \mathrm{min}^{-1}$ pump flow. Total systemic flow is not known for that phase but could be expected to be much higher. Mixed venous oxygen tension corresponded with mixed venous oxygen saturation, decreasing with decreasing flow.

The relationship of resting mixed venous oxygen saturation with the hemoglobin concentration is shown in Table II, with the data arbitrarily divided into three distinct groups. Mixed venous oxygen saturation at higher serum hemoglobin levels (mean $9.1 \pm 0.1 \mathrm{gm} / \mathrm{dl}$ ) was $46.5 \% \pm 1.7 \%$, which was significantly higher. The hemoglobin concentration was not artificially manipulated, and arterial oxygen saturation was always greater than $95 \%$ with the animal breathing room air. There were no significant differences in body temperature, pump flow rate, or oxygen consumption among three groups.

No differences in total catecholamine concentration were noted at 100 and $120 \mathrm{ml} \cdot \mathrm{kg}^{-1} \cdot \mathrm{min}^{-1}$ 
Table I. Blood gas analysis at rest in calves with chronic nonpulsatile biventricular bypass

\begin{tabular}{cccccc}
\hline $\begin{array}{c}N F R \\
\left(\mathrm{ml} \cdot \mathrm{kg}^{-1} \cdot \mathrm{min}^{-1}\right)\end{array}$ & $\begin{array}{c}A F R \\
\left(\mathrm{ml} \cdot \mathrm{kg}^{-1} \cdot \mathrm{min}^{-1}\right)\end{array}$ & $\begin{array}{c}\text { Blood pressure } \\
(\mathrm{mm} \mathrm{Hg})\end{array}$ & $\begin{array}{c}\mathrm{Hb} \\
(\mathrm{gm} / \mathrm{dl})\end{array}$ & $\begin{array}{c}\mathrm{SaO}_{2} \\
(\%)\end{array}$ & $\begin{array}{c}\mathrm{PvO}_{2} \\
(\mathrm{~mm} \mathrm{Hg})\end{array}$ \\
\hline $90(n=27)$ & $88 \pm 1$ & $95.2 \pm 1.7$ & $8.23 \pm 0.11$ & $97.0 \pm 0.5$ & $23.8 \pm 0.9^{*}$ \\
$100(n=28)$ & $102 \pm 1$ & $97.0 \pm 1.7$ & $7.92 \pm 0.17$ & $97.7 \pm 0.6$ & $28.3 \pm 1.2 \neq$ \\
$120(n=22)$ & $118 \pm 2$ & $92.9 \pm 2.4$ & $8.43 \pm 0.16$ & $96.0 \pm 0.8$ & $29.6 \pm 1.0$ \\
\hline
\end{tabular}

Data are mean \pm standard error of the mean. $N R F$, Nominal left pump flow rate; $A F R$, actual left pump flow rate; $\mathrm{Hb}$, serum hemoglobin level; $\mathrm{SaO}_{2}$, arterial oxygen saturation; $\mathrm{P \nu O}_{2}$, mixed venous oxygen tension; $\mathrm{SvO}_{2}$, mixed venous oxygen saturation, $\mathrm{DO}_{2}$, oxygen delivery.

${ }^{*} p<0.01 \mathrm{vs} 120 \mathrm{ml} \cdot \mathrm{kg}^{-1} \cdot \mathrm{min}^{-1}$, according to analysis of variance, Scheffe $F$ test.

$\dagger p<0.05 \mathrm{vs} 100 \mathrm{ml} \cdot \mathrm{kg}^{-1} \cdot \mathrm{min}^{-1}$, according to analysis of variance, Scheffe $F$ test

$t p<0.05 \mathrm{vs} 90 \mathrm{ml} \cdot \mathrm{kg}^{-1} \cdot \mathrm{min}^{-1}$, according to analysis of variance, Scheffe $F$ test.

$\$ p<0.01 \mathrm{vs} 120 \mathrm{ml} \cdot \mathrm{kg}^{-1} \cdot \mathrm{min}^{-1}$, according to analysis of variance, Scheffe $F$ test

Table II. Effects of hemoglobin concentration on oxygen transport in claves with chronic nonpulsatile biventricular bypass

\begin{tabular}{|c|c|c|c|c|c|c|c|c|c|}
\hline$H b(\mathrm{gm} / \mathrm{dl})$ & $\begin{array}{c}\text { Mean } \mathrm{Hb} \\
(\mathrm{gm} / \mathrm{dl})\end{array}$ & $\begin{array}{c}B T \\
\left({ }^{\circ} \mathrm{C}\right)\end{array}$ & $\begin{array}{c}\text { Flow } \\
\left(\mathrm{ml} \cdot \mathrm{kg}^{-1}\right. \\
\left.\mathrm{min}^{-1}\right)\end{array}$ & $\begin{array}{l}\mathrm{SaO}_{2} \\
(\%)\end{array}$ & $\begin{array}{c}\mathrm{PvO}_{2} \\
(\mathrm{~mm} \mathrm{Hg})\end{array}$ & $\begin{array}{l}\mathrm{SvO}_{2} \\
(\%)\end{array}$ & $\begin{array}{c}\text { Oxygen } \\
\text { extraction } \\
(\%)\end{array}$ & $\begin{array}{c}\mathrm{VO}_{2} \\
\left(\mathrm{ml} \cdot \mathrm{kg}^{-1}\right. \\
\left.\mathrm{min}^{-1}\right)\end{array}$ & $\begin{array}{c}\mathrm{Do}_{2} \\
\left(\mathrm{ml} \cdot \mathrm{kg}^{-1}\right. \\
\left.\mathrm{min}^{-1}\right)\end{array}$ \\
\hline$<7.5(n=24)$ & $7.21 \pm 0.06$ & $39.2 \pm 0.1$ & \pm 2 & $96.7 \pm 0.7$ & $25.9 \pm 0.09$ & $38.8 \pm 1.9$ & $59.2 \pm 2.1$ & $6.24 \pm 0.27$ & $9.99 \pm 0.27$ \\
\hline $\begin{array}{c}7.6>\mathrm{Hb}>8.4 \\
(n=29)\end{array}$ & $8.16 \pm 0.05$ & $39.1 \pm 0.1$ & & $97.2 \pm 0.4$ & $26.1 \pm 1.0$ & $40.4 \pm 2.0$ & $57.8 \pm 2.1$ & $6.35 \pm 0.20$ & $10.94 \pm 0.30$ \\
\hline$>8.4(n=24)$ & $9.09 \pm 0.09$ & $39.3 \pm 0.1$ & $106 \pm 2$ & $96.8 \pm 0.08$ & $29.6 \pm 1.2$ & $46.5 \pm 1.7^{*}$ & $51.2 \pm 1.9^{*}$ & $6.68 \pm 0.25$ & $12.90 \pm 0.36 \dagger$ \\
\hline
\end{tabular}

Data are mean \pm standard error of the mean. $\mathrm{Hb}$. Hemoglobin level; $B T_{x}$ body temperature (rectal); $\mathrm{SaO}_{2}$, arterial oxygen saturation; $P \mathrm{vo}_{2}$, mixed venous oxygen tension; $\mathrm{SvO}_{2}$, mixed venous oxygen saturation; $\mathrm{DO}_{2}$, oxygen delivery.

${ }^{*} p<0.05 \mathrm{vs} \mathrm{Hb}<7.5$, according to analysis of variance, Scheffe $F$ test.

$\dagger p<0.01$ vs $\mathrm{Hb}<7.5$ and $7.6<\mathrm{Hb}<8.4$, according to analysis of variance, Scheffe $F$ test.

Table III. Effects of pump flow rate on serum catecholamine levels during chronic nonpulsatile biventricular bypass

\begin{tabular}{|c|c|c|c|c|}
\hline NFR $\left(\mathrm{ml} \cdot \mathrm{kg}^{-1} \cdot \min ^{-1}\right)$ & $\begin{array}{l}\text { Norepinephrine } \\
(\mathrm{gg} / \mathrm{ml})\end{array}$ & $\begin{array}{l}\text { Epinephrine } \\
(\mathrm{pg} / \mathrm{ml})\end{array}$ & $\begin{array}{c}\text { Dopamine } \\
(\mathrm{pg} / \mathrm{ml})\end{array}$ & $\begin{array}{l}\text { TOTAL } \\
(\mathrm{pg} / \mathrm{ml})\end{array}$ \\
\hline $90(n=5)$ & $1065 \pm 179^{*}$ & $243 \pm 132$ & $16 \pm \overline{16}$ & $1324 \pm 274^{*}$ \\
\hline 100 and $120(n=10)$ & $467 \pm 102$ & $62 \pm 21$ & $2 \pm 6$ & $532 \pm 111$ \\
\hline Before operation $(n=3)$ & $267 \pm 5$ & $45 \pm 39$ & $10 \pm 14$ & $309 \pm 61$ \\
\hline
\end{tabular}

Data are mean \pm standard error of the mean. $N R F$, Nominal left pump flow rate.

${ }^{*} p<0.01$ vs 100 and $120 \mathrm{ml} \cdot \mathrm{kg}^{-1} \cdot \mathrm{min}^{-1}$, according to unpaired $t$ test.

pump flow rates (Table III). There was a significant $(p<0.01$, unpaired $t$ test $)$ increase in total catecholamine level at $90 \mathrm{ml} \cdot \mathrm{kg}^{-1} \cdot \min ^{-1}$ of pump flow. Significant $(p<0.05)$ correlation between serum catecholamine concentration and oxygen extraction rate was also observed (Fig. 2), with norepinephrine being the major component of the increase in total catecholamine level at $90 \mathrm{ml} \cdot \mathrm{kg}^{-1} \cdot \mathrm{min}^{-1}$ of pump flow rate (Table III).

During exercise. The correlation between maximal exercise capacity and pump flow rate is shown in Tables IV, V, and VI. The maximal treadmill time that the animals achieved at a pump flow rate of 90 $\mathrm{ml} \cdot \mathrm{kg}^{-1} \cdot \min ^{-1}$ was significantly lower than that achieved at other nominal flow rates, but there was no significant difference in animal exercise tolerance between pump flow rates of 100 and 120 $\mathrm{ml} \cdot \mathrm{kg}^{-1} \cdot \min ^{-1}$.

Blood gas analyses at each nominal flow rate are also shown in Tables IV through VI. Oxygen delivery, calculated from arterial oxygen content and pump flow rate, increased gradually during progressive exercise and differed significantly among the three nominal flow rates. $\dot{\mathrm{V}}_{2}$ also increased significantly with exercise in accordance with increases in workload, as did oxygen extraction. $\dot{\mathrm{VO}}_{2}$ was significantly higher at a nominal pump flow rate of $120 \mathrm{ml} \cdot \mathrm{kg}^{-1} \cdot \min ^{-1}$ than at other flow rates at treadmill speeds of 0.7 and $0.9 \mathrm{mph}$ and at 30 minutes after exercise. At a nominal flow rate of 
Table I. Continued

\begin{tabular}{|c|c|c|c|c|}
\hline $\mathrm{SvO}_{2}(\%)$ & $\begin{array}{c}\text { Oxygen } \\
\text { extraction (\%) }\end{array}$ & $\left(\mathrm{ml} \cdot \mathrm{kg}^{-1} \cdot \mathrm{min}^{-1}\right)$ & $\begin{array}{c}\mathrm{Do}_{2} \\
\left(\mathrm{ml} \cdot \mathrm{kg}^{-1} \cdot m i n^{-1}\right)\end{array}$ & $\begin{array}{l}\text { Lactate } \\
(m E q / L)\end{array}$ \\
\hline $35.9 \pm 2.0^{*}$ & $62.3 \pm 0.21^{*}$ & $6.26 \pm 0.26$ & $9.69 \pm 0.23 \dagger$ & $7.81 \pm 2.42 \dagger$ \\
\hline $43.0 \pm 1.6+$ & $55.2 \pm 1.7 \div$ & $6.15 \pm 0.17$ & $10.97 \pm 0.24 \S$ & $0.71 \pm 0.06$ \\
\hline $46.8 \pm 1.9$ & $50.7 \pm 1.9$ & $6.85 \pm 0.25$ & $13.34 \pm 0.35$ & $0.73 \pm 0.27$ \\
\hline
\end{tabular}

Table IV. Blood gas analysis during treadmill exercise in calves with chronic centrifugal biventricular bypass at pump flow rate before exercise of $90 \mathrm{ml} \cdot \mathrm{kg}^{-1} \cdot \mathrm{min}^{-1}$

\begin{tabular}{|c|c|c|c|c|c|c|c|c|c|}
\hline $\begin{array}{c}\text { Treadmill } \\
\text { speed } \\
\text { (mph) }\end{array}$ & $n$ & $\begin{array}{l}\mathrm{SaO}_{2} \\
(\%)\end{array}$ & $\begin{array}{c}\mathrm{PvO}_{2} \\
(\mathrm{~mm} \mathrm{Hg})\end{array}$ & $\begin{array}{l}\mathrm{SVO}_{2} \\
(\%)\end{array}$ & $\begin{array}{c}\mathrm{Do}_{2} \\
\left(\mathrm{ml} \cdot \mathrm{kg}^{-1}\right. \\
\left.\mathrm{min}^{-1}\right)\end{array}$ & $\begin{array}{l}\mathrm{A}-\mathrm{VO}_{2} \\
(\mathrm{ml} / \mathrm{dl})\end{array}$ & $\begin{array}{c}\text { Oxygen } \\
\text { extraction } \\
(\%)\end{array}$ & $\begin{array}{c}\dot{\dot{V O}_{2}} \\
\left(\mathrm{ml} \cdot \mathrm{kg}^{-1}\right. \\
\left.\min ^{-1}\right)\end{array}$ & $\begin{array}{l}\text { Lactate } \\
(m E q / L)\end{array}$ \\
\hline 0 (before) & 10 & $96.6 \pm 0.9$ & $21.2 \pm 1.2$ & $29.2 \pm 1.2$ & $9.86 \pm 0.38$ & $7.91 \pm 0.59$ & $69.8 \pm 3.4$ & $6.96 \pm 0.55$ & $7.17 \pm 2.26$ \\
\hline 0.3 & 10 & $96.1 \pm 0.7$ & $17.1 \pm 0.8^{*}$ & $20.4 \pm 1.8 \dagger$ & $9.88 \pm 0.33$ & $8.81 \pm 0.45^{*}$ & $78.7 \pm 1.9 \dagger$ & $7.80 \pm 0.38 \ddagger$ & $8.19 \pm 2.40$ \\
\hline 0.5 & 5 & $92.0 \pm 2.9$ & $17.4 \pm 0.4 \dagger$ & $20.7 \pm 2.0 \ddagger$ & $9.27 \pm 0.40$ & $7.90 \pm 0.28$ & $77.4 \pm 2.1 \mp$ & $7.16 \pm 0.28$ & $1.45 \pm 0.18$ \\
\hline 0.7 & 4 & $90.2 \pm 3.8$ & $16.5 \pm 0.9$ & $17.9 \pm 1.3^{*}$ & $9.15 \pm 0.74$ & $7.95 \pm 0.65^{*}$ & $80.0 \pm 1.7 \S$ & $7.34 \pm 0.68 \pm$ & $1.47 \pm 0.28$ \\
\hline 0.9 & 3 & $92.3 \pm 3.1$ & $15.7 \pm 0.9^{*}$ & $17.2 \pm 2.0^{*}$ & $9.38 \pm 0.41$ & $8.42 \pm 0.48^{*}$ & $81.2 \pm 2.9 \S$ & $7.63 \pm 0.51 \neq$ & $2.15 \pm 0.69$ \\
\hline 1.1 & 3 & $95.5 \pm 0.1$ & $16.0 \pm 1.5 \dagger$ & $16.6 \pm 1.6 \dagger$ & $9.73 \pm 0.50 \ddagger$ & $8.89 \pm 0.44^{*}$ & $82.7 \pm 1.7 \dagger$ & $8.05 \pm 0.46 \neq$ & $2.90 \pm 1.14$ \\
\hline 0 (after) & 10 & $96.3 \pm 0.8$ & $22.1 \pm 0.9$ & $29.5 \pm 1.7$ & $9.55 \pm 0.34$ & $7.78 \pm 0.31$ & $69.5 \pm 1.7$ & $6.64 \pm 0.28$ & $8.32 \pm 2.08$ \\
\hline
\end{tabular}

Data mean \pm standard error of the mean. $\mathrm{SaO}_{2}$, Arterial oxygen saturation; $\mathrm{PvO}_{2}$, mixed venous oxygen tension; $\mathrm{SvO}_{2}$, mixed venous oxygen saturation, $\mathrm{DO}_{2}$, oxygen delivery; $\mathrm{A}-\mathrm{VO}_{2}$, arteriovenous oxygen content difference.

$* p<0.005$ versus preexercise value, according to paired $t$ test.

$\dagger p<0.01$ versus preexercise value, according to paired $t$ test.

$\uparrow p<0.05$ versus preexercise value, according to paired $t$ test.

$\$ p<0.001$ versus preexercise value, according to paired $t$ test.

$90 \mathrm{ml} \cdot \mathrm{kg}^{-1} \cdot \min ^{-1}$, the mixed venous oxygen tension was significantly lower than at other nominal flow rates before exercise, at $0.3,0.5,0.7$, and 0.9 mph of treadmill speed, and at 30 minutes after exercise. There were no significant differences in mixed venous oxygen tension or arteriovenous oxygen at nominal flow rates of 100 and 120 $\mathrm{ml} \cdot \mathrm{kg}^{-1} \cdot \min ^{-1}$.

Progressive increase in muscular work was expressed as a percentage of maximal oxygen uptake $\dot{\mathrm{V}}_{2}\left(\dot{\mathrm{VO}}_{2 \text { max }}\right)$. The incremental responses in oxygen extraction at the three nominal flow rates were significant and linear, and there were no significant differences among three nominal flow rates (Fig. 3). The maximal values of oxygen extraction were greater than $70 \%$ at each nominal flow rate, and more than $70 \%$ of the maximal oxygen extraction occurred at $85 \%$ of the maximal oxygen uptake. The mixed venous oxygen saturation also correlated significantly with $\dot{\mathrm{V}}_{2}$ max and decreased to approximately $20 \%$ at the maximal oxygen uptake for each flow rate. The serum lactate concentration was increased at $90 \%$ of $\dot{\mathrm{VO}}_{2}$ max at a nominal flow rate of $100 \mathrm{ml} \cdot \mathrm{kg}^{-1} \cdot \mathrm{min}^{-1}$ and at $100 \%$ of maximum $\dot{V}_{\mathrm{O}_{2}}$ at $120 \mathrm{ml} \cdot \mathrm{kg}^{-1} \cdot \mathrm{min}^{-1}$, but there were no significant differences between the two groups. At a nominal flow rate of $90 \mathrm{ml} \cdot \mathrm{kg}^{-1} \cdot \mathrm{min}^{-1}$, the lactate level was abnormally high even before exercise (Table IV). The relationship between $\dot{\mathrm{VO}}_{2}$ max and oxygen delivery obtained from each nominal flow rate was a straight line near the origin with a slope of 9.78 (Fig. 4).

At autopsy, all calves showed lung adhesions and mild atelectasis at the site of operation. Although some small renal infarcts were seen, there was no significant pathology that would have had a major effect on our investigation.

\section{Discussion}

A number of acute studies have indicated the superiority of pulsatile over nonpulsatile cardiopulmonary bypass during operation on the basis of significant increases in oxygen consumption. ${ }^{12-14}$ Other studies, ${ }^{15,16}$ however, have demonstrated no significant differences in oxygen consumpton between the two different flow patterns. This investigation used a chronic nonpulsatile animal model to determine whether these differences were also present in the chronic state. Unique features of this model and protocol were as follows: (1) The animal was fully alert and well past the operative day. (2) Fixed flow levels were set. (3) The test flow levels 
Table V. Blood gas analysis during treadmill exercise in calves with chronic centrifugal biventricular bypass at pump flow rate before exercise of $100 \mathrm{ml} \cdot \mathrm{kg}^{-1} \cdot \mathrm{min}^{-1}$

\begin{tabular}{|c|c|c|c|c|c|c|c|c|c|}
\hline $\begin{array}{c}\text { Treadmill } \\
\text { speed } \\
\text { (mph) }\end{array}$ & $n$ & $\begin{array}{l}\mathrm{SaO}_{2} \\
(\%)\end{array}$ & $\begin{array}{c}\mathrm{PvO}_{2} \\
(\mathrm{~mm} \mathrm{Hg})\end{array}$ & $\begin{array}{l}\mathrm{SvO}_{2} \\
(\%)\end{array}$ & $\begin{array}{c}\mathrm{Do}_{2} \\
\left(\mathrm{ml} \cdot \mathrm{kg}^{-1}\right. \\
\left.\mathrm{min}^{-1}\right)\end{array}$ & $\begin{array}{l}\mathrm{A}-\mathrm{VO}_{2} \\
(\mathrm{ml} / \mathrm{dl})\end{array}$ & $\begin{array}{c}\text { Oxygen } \\
\text { extraction } \\
(\%)\end{array}$ & $\begin{array}{c}\dot{\mathrm{VO}_{2}} \\
\left(\mathrm{ml} \cdot \mathrm{kg}^{-1}\right. \\
\left.m i n^{-1}\right)\end{array}$ & $\begin{array}{c}\text { Lactate } \\
(m E q / L)\end{array}$ \\
\hline 0 (before) & 10 & $93.7 \pm 0.7$ & $28.5 \pm 2.1$ & $42.8 \pm 3.3$ & $11.8 \pm 0.5$ & $6.42 \pm 0.30$ & $55.9 \pm 3.6$ & $6.50 \pm 0.28$ & $1.00 \pm 0.09$ \\
\hline 0.3 & 10 & $97.6 \pm 0.8$ & $1.6 \pm 1.8^{*}$ & $33.0 \pm 2$ & $12.4 \pm 0.5 \%$ & $7.65 \pm 0.25 \dagger$ & $66.2 \pm 2.8 \dagger$ & $7.96 \pm 0.29^{*}$ & 0.06 \\
\hline 0.5 & 10 & $97.2 \pm 0.9$ & $23.2 \pm 1.7^{*}$ & $28.7 \pm 2.9^{*}$ & $12.1 \pm 0.5$ & $8.07 \pm 0.29^{*}$ & $70.4 \pm 3.2^{*}$ & $8.43 \pm 0.33^{*}$ & \pm 0.11 \\
\hline 0.7 & 10 & $96.7 \pm 0.7$ & $22.5 \pm 1.6^{*}$ & $27.3 \pm 2.5^{*}$ & $12.1 \pm 0.5$ & $8.22 \pm 0.3$ & $71.8 \pm 2$ & $8.62 \pm 0.34 *$ & $=0.15$ \\
\hline 0.9 & 9 & $97.0 \pm 0.08$ & $22.3 \pm 1.6^{*}$ & $26.3 \pm 2.5^{*}$ & $12.2 \pm 0.6$ & $8.40 \pm 0.33^{*}$ & $72.9 \pm 2.7^{*}$ & $8.85 \pm 0.36^{*}$ & 0.83 \\
\hline 1.1 & 8 & $96.7 \pm 0.9$ & $21.0 \pm 1.8$ & $21.8 \pm 2.7^{*}$ & $12.3 \pm 0.7$ & $8.80 \pm 0.38 *$ & $77.4 \pm 2.9 *$ & $9.42 \pm 0.47^{*}$ & $1.73 \pm 0.59$ \\
\hline 1.3 & 7 & $96.8 \pm 0.9$ & $20.3 \pm 1.2 \ddagger$ & $23.4 \pm 2.5 \S$ & $12.2 \pm 0.8$ & $8.62 \pm 0.52 \S$ & $75.9 \pm 2.6 \S$ & $9.25 \pm 0.58 \dagger$ & $1.75 \pm 0.55$ \\
\hline 1.5 & 6 & $95.9 \pm 1.0$ & $19.0 \pm 0.9 \ddagger$ & $22.3 \pm 2.2 \S$ & $13.0 \pm 0.9$ & $9.37 \pm 0.65$ & $76.6 \pm 2.5 \S$ & $9.99 \pm 0.81 \S$ & $2.26 \pm 0.88$ \\
\hline 0 (after) & 10 & $97.8 \pm 0.7$ & $28.4 \pm 1.5$ & $29.5 \pm 1.7$ & $12.1 \pm 0.5$ & $6.49 \pm 0.25$ & $54.4 \pm 2.7$ & $6.50 \pm 0.25$ & $1.26 \pm 0.29$ \\
\hline
\end{tabular}

Data mean \pm standard error of the mean. $\mathrm{SaO}_{2}$, Arterial oxygen saturation; $\mathrm{PvO}_{2}$, mixed venous oxygen tension; $\mathrm{SvO}_{2}$, mixed venous oxygen saturation; $\mathrm{DO}_{2}$, oxygen delivery; $\mathrm{A}-\mathrm{VO}_{2}$, arteriovenous oxygen content difference.

${ }^{*} p<0.001$ versus preexercise value, according to paired $t$ test.

$t p<0.005$ versus preexercise value, according to paired $t$ test.

$\ddagger p<0.05$ versus preexercise value, according to paired $t$ test.

$\$ p<0.01$ versus preexercise value, according to paired $t$ test.

Table VI. Blood gas analysis during treadmill exercise in calves with chronic centrifugal biventricular bypass at pump flow rate before exercise of $120 \mathrm{ml} \cdot \mathrm{kg}^{-1} \cdot \mathrm{min}^{-1}$

\begin{tabular}{|c|c|c|c|c|c|c|c|c|c|}
\hline $\begin{array}{c}\text { Treadmill } \\
\text { speed } \\
(\text { mph })\end{array}$ & $n$ & $\begin{array}{c}\mathrm{SaO}_{2} \\
(\%)\end{array}$ & $\begin{array}{c}\mathrm{PvO}_{2} \\
(\mathrm{~mm} \stackrel{\mathrm{H} g}{ })\end{array}$ & $\begin{array}{l}\mathrm{SVO}_{2} \\
(\%)\end{array}$ & $\begin{array}{c}\mathrm{DO}_{2} \\
\left(\mathrm{ml} \cdot \mathrm{kg}^{-1}\right. \\
\left.\mathrm{min}^{-1}\right)\end{array}$ & $\begin{array}{l}\mathrm{A}-\mathrm{VO}_{2} \\
(\mathrm{ml} / \mathrm{dl})\end{array}$ & $\begin{array}{c}\text { Oxygen } \\
\text { extraction } \\
(\%)\end{array}$ & $\begin{array}{c}\mathrm{VO}_{2} \\
\left(\mathrm{ml} \cdot \mathrm{kg}^{-1}\right. \\
\left.\mathrm{min}^{-1}\right)\end{array}$ & $\begin{array}{l}\text { Lactate } \\
(m E q / L)\end{array}$ \\
\hline 0 (before) & 10 & $97.3 \pm 1.1$ & $28.1 \pm 2.2$ & $43.3 \pm 3.5$ & $13.9 \pm 0.4$ & $6.38 \pm 0.29$ & $55.9 \pm 3.4$ & $7.58 \pm 0.31$ & $0.99 \pm 0.21$ \\
\hline 0.3 & 10 & $98.3 \pm 0.9$ & $25.6 \pm 2.0^{*}$ & $37.2 \pm 3.2 \dagger$ & $14.2 \pm 0.4$ & $7.22 \pm 0.32 \ddagger$ & $62.3 \pm 3.2 \dagger$ & $8.77 \pm 0.34 \ddagger$ & $1.05 \pm 0.31$ \\
\hline 0.5 & 10 & $97.2 \pm 1.1$ & $24.9 \pm 2.0 \ddagger$ & $34.3 \pm 3.7 \ddagger$ & $14.0 \pm 0.4$ & $7.38 \pm 0.336 \div$ & $64.8 \pm 3.7$ & $8.99 \pm 0.35 \ddagger$ & $0.85 \pm 0.22$ \\
\hline 0.7 & 9 & $97.6 \pm 0.9$ & $23.3 \pm 1.8 \ddagger$ & $29.8 \pm 3.6 \ddagger$ & $14.3 \pm 0.5$ & $7.95 \pm 0.30 \ddagger$ & $69.5 \pm 3.6 \div$ & $9.78 \pm 0.30 \div$ & $1.03 \pm 0.28$ \\
\hline 0.9 & 7 & $97.7 \pm 0.9$ & $21.4 \pm 2.2 \ddagger$ & $25.3 \pm 3.9 \ddagger$ & $14.3 \pm 0.6$ & $8.49 \pm 0.33 \ddagger$ & $74.2 \pm 3.9 \pm$ & $10.47 \pm 0.33 \ddagger$ & $1.06 \pm 0.25$ \\
\hline 1.1 & 7 & $97.6 \pm 1.0$ & $20.6 \pm 2.2 \dagger$ & $23.8 \pm 3.6 \ddagger$ & $14.6 \pm 0.5 \S$ & $8.75 \pm 0.35 \ddagger$ & $75.5 \pm 3.8 \ddagger$ & $10.85 \pm 0.31 \dagger$ & $1.16 \pm 0.25$ \\
\hline 1.3 & 6 & $96.6 \pm 1.0$ & $19.0 \pm 1.4^{*}$ & $23.1 \pm 4.2^{*}$ & $14.2 \pm 0.7$ & $8.67 \pm 0.54^{*}$ & $76.0 \pm 4.5^{*}$ & $10.67 \pm 0.58^{*}$ & $1.10 \pm 0.29$ \\
\hline 1.5 & 5 & $96.7 \pm 1.1$ & $19.0 \pm 1.3^{*}$ & $19.2 \pm 4.2 \dagger$ & $14.7 \pm 0.6$ & $9.34 \pm 0.35 \dagger$ & $80.0 \pm 4.5 \dagger$ & $11.53 \pm 0.31$ & $2.04 \pm 0.68$ \\
\hline 0 (after) & 10 & $96.7 \pm 0.8$ & $27.6 \pm 1.7$ & $40.6 \pm 2.8$ & $13.6 \pm 0.4$ & $6.57 \pm 0.24$ & $58.1 \pm 2.8$ & $7.91 \pm 0.29$ & $1.20 \pm 0.35$ \\
\hline
\end{tabular}

Data mean \pm standard error of the mean. $\mathrm{SaO}_{2}$, Arterial oxygen saturation; $\mathrm{P}_{\mathrm{vO}}$, mixed venous oxygen tension; $\mathrm{SvO}_{2}$, mixed venous oxygen saturation; $\mathrm{DO}_{2}$, oxygen delivery; $\mathrm{A}-\mathrm{YO}_{2}$, arteriovenous oxygen content difference.

${ }^{*} p<0.001$ versus preexercise value, according to paired $t$ test.

$\dagger p<0.005$ versus preexercise value, according to paired $t$ test.

$t p<0.01$ versus preexercise value, according to paired $t$ test.

$\$ p<0.05$ versus preexercise value, according to paired $t$ test.

were maintained for 7 days each. (4) The natural heart ventricle was fibrillated, so that flow was exclusively provided and controlled by the nonpulsatile centrifugal pump.

Resting oxygen consumption. Lunn and coworkers ${ }^{17}$ made determinations of resting $\dot{\mathrm{V}}_{2}$ in calves used for pulsatile artificial heart implantations and obtained values of 5.79 (before operation), 9.0 (2 weeks after operation), 7.03 ( 3 weeks after operation), and $5.59 \mathrm{ml} \cdot \mathrm{kg}^{-1} \cdot \mathrm{min}^{-1}$ (4 weeks after operation). Shimomitsu and associates ${ }^{18}$ reported a much lower $4.44 \mathrm{ml} \cdot \mathrm{kg}^{-1} \cdot \mathrm{min}^{-1}$ in calves with total artificial hearts but these were much larger and older animals. Younger specimens are known to have a higher level of metabolism than mature animals. ${ }^{19}$ Our results were in the range of 6.7 $\mathrm{ml} \cdot \mathrm{kg}^{-1} \cdot \mathrm{min}^{-1}$.

These calves consistently maintained an oxygen saturation of $96 \%$ to $98 \%$. Neither the pump flow level nor the nonpulsatility seemed to affect the systemic arterial oxygen saturation. This is consistent with the results of Hauge and Nicolaysen, ${ }^{20}$ who saw no difference between steady and pulsatile flow with respect to the function of normal lungs. 


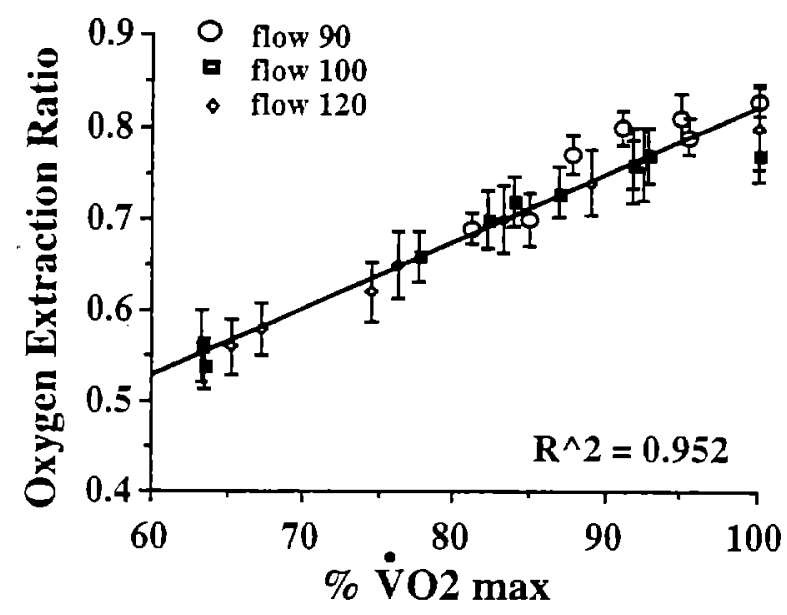

Fig. 3. Relationship between oxygen extraction and $\dot{\mathrm{V}}_{2}$ max. Oxygen extraction correlated significantly $(p<0.01)$ with percentage of $\mathrm{VO}_{2}$ max ; flow $90,90 \mathrm{ml} \cdot \mathrm{kg}^{-1} \cdot \mathrm{min}^{-1} ;$ flow $100,100 \mathrm{ml} \cdot \mathrm{kg}^{-1} \cdot \mathrm{min}^{-1}$; flow $120,120 \mathrm{ml} \cdot \mathrm{kg}^{-1} \cdot \mathrm{min}^{-1}$.

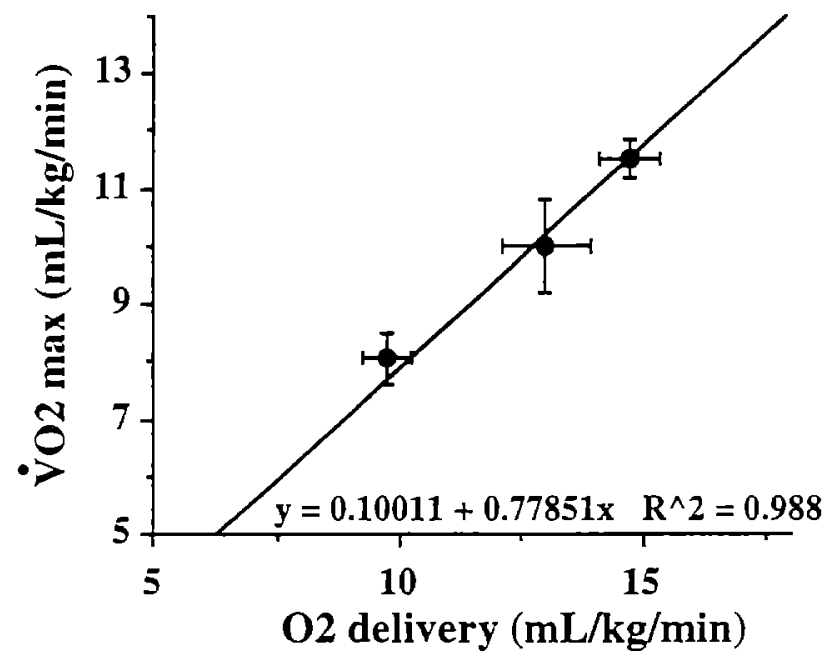

Fig. 4. The correlation between $\mathrm{VO}_{2}$ max and oxygen delivery. Each data point was derived from the $\dot{\mathrm{VO}}_{2 \text { max }}$ to oxygen delivery ratio obtained at each nominal pump flow rate. Small bars indicate standard error of the mean. $\dot{V}_{2}$ max correlated significantly with oxygen delivery. The slope of the correlation line is approximately 0.78 .

At a nominal perfusion of $90 \mathrm{ml} \cdot \mathrm{kg}^{-1} \cdot \mathrm{min}^{-1}$, the calves clearly had a major change in serum lactate level with respect to the other flow conditions, indicating anaerobic metabolism, and oxygen delivery to the tissues was insufficient to maintain normal organ function. Either oxygen delivery, oxygen extraction, or both were therefore inadequate in this nonpulsatile blood flow condition.

The normal range of mixed venous oxygen tension in the resting calf has been reported to be
25.7 to $39.7 \mathrm{~mm} \mathrm{Hg}$ (mean 32.7) by Nelson, Ekins, and Allen. ${ }^{21}$ Lunn and colleagues ${ }^{17}$ documented $38 \pm 4$ $\mathrm{mm} \mathrm{Hg}$ ( \pm standard deviation). Kasnitz and cowork$\mathrm{ers}^{22}$ reported the relationship between mixed venous oxygen tension and serum lactate concentration in 20 patients with severe cardiac or pulmonary disease and showed that mixed venous oxygen tension correlated better with both hyperlactatemia and survival than did cardiac output or arterial oxygen tension. A mixed venous oxygen tension of less than 28 
$\mathrm{mm} \mathrm{Hg}$ was associated with hyperlactatemia and was always associated with death in their study. Simmons and associates ${ }^{23}$ reported that the lactate production threshold for mixed venous oxygen tension was $27 \mathrm{~mm}$ $\mathrm{Hg}$ in dogs under conditions of arterial hypoxia, low cardiac output, or both. These results suggest that when vasoregulation is intact, there is a threshold value or critical mixed venous oxygen tension of about $28 \mathrm{~mm} \mathrm{Hg}$ below which anaerobic metabolism is usually manifested as an increase in blood lactate. The calves in our study averaged a mixed venous oxygen tension of $23.8 \mathrm{~mm} \mathrm{Hg}$ at $90 \mathrm{ml} \cdot \mathrm{kg}^{-1} \cdot \mathrm{min}^{-1}$, rising to 28.3 at $100 \mathrm{ml} \cdot \mathrm{kg}^{-1} \cdot \mathrm{min}^{-1}$.

At $90 \mathrm{ml} \cdot \mathrm{kg}^{-1} \cdot \mathrm{min}^{-1}$ perfusion, oxygen delivery was about $10 \mathrm{ml} \cdot \mathrm{kg}^{-1} \cdot \mathrm{min}^{-1}$, whereas the next higher flow supplied $11 \mathrm{ml} \cdot \mathrm{kg}^{-1} \cdot \mathrm{min}^{-1}$ of oxygen. On the evidence of the lactate level and mixed venous oxygen tension the critical point of oxygen delivery exists between 10 and $11 \mathrm{ml}$. $\mathrm{kg}^{-1} \cdot \min ^{-1}$, as found by a number of researchers. ${ }^{24-29}$ The value in our study appears to be high, but anesthesia, mechanical ventilation, and paralyzing agents, present in most of these previous studies, are known to decrease oxygen demand. It must be noted that the calves we used were much younger than most of the other test animals and would therefore be expected to have a more active metabolism. Further, the critical oxygen delivery is sensitive to the method used to calculate it, and our current chronic approach is unique. Lambs studied by Heusser, Fahey, and Lister ${ }^{27}$ were younger still and only sedated for testing, the instrumentation having been previously implanted and their critical values for systemic oxygen transport defined by a $\mathrm{V}_{\mathrm{O}_{2}}$ method similar to ours. Without similarly obtained chronic data from pulsatile animal models, firm and final conclusions are not possible, but the possibility remains that a slightly higher critical oxygen delivery is required when the blood flow is steady rather than pulsing.

Heusser, Fahey, and Lister ${ }^{27}$ showed oxygen extraction at the critical point to be $60 \%$ to $65 \%$, whereas Schumacker, Long, and Wood ${ }^{30}$ found it to be $73 \%$ and Pepe and Culver ${ }^{28}$ found it to be only $50 \%$. If the critical pump flow in our experiments is considered to be $100 \mathrm{ml} \cdot \mathrm{kg}^{-1} \cdot \mathrm{min}^{-1}$, the critical extraction ratio would be $55 \%$, a low but not unprecedented level.

Exercise and oxygen utilization. An advantage of this awake, chronic, nonpulsatile blood flow preparation is the opportunity to study responses to the stress of exercise. Wilkens, Regelson, and Hoffmeis- ter $^{31}$ suggested that nonpulsatile flow produces stagnation of the microcirculation, opens arteriovenous shunts, and results in poor lymphatic flow and edema. Ogata and associates ${ }^{32}$ showed an increase in omental volume during nonpulsatile flow, suggesting stagnation of lymph and tissue edema. Because this would increase the capillary-tissue distances and diffusion through tissue is known to be a limitation to oxygen utilization, ${ }^{33}$ nonpulsatile blood flow could be expected to limit oxygen delivery and extraction during exercise.

Roca and coworkers ${ }^{34}$ demonstrated evidence for tissue diffusion limitation of $\mathrm{VO}_{2 \text { max }}$ in normal humans. In their study, oxygen consumption was measured at maximal exercise in athletes at inspired oxygen gas concentrations between $12 \%$ and $21 \%$. The relationship between $\dot{\mathrm{VO}}_{2}$ and oxygen delivery was noted to be a straight line through the origin, indicating that $\dot{\mathrm{V}}_{2}$ max was $75 \%$ to $80 \%$ of oxygen delivery. Weber and colleagues ${ }^{35}$ and Weber and Janicki ${ }^{36}$ measured cardiac output, systemic oxygen extraction, and lactate production during progressive exercise in patients with chronic heart failure. Their patients demonstrated $70 \%$ to $75 \%$ of maximal oxygen extraction at more than $80 \%$ of $\mathrm{VO}_{2}$ max. Wilson and coworkers ${ }^{37}$ also demonstrated $74 \%$ to $83 \%$ of maximal systemic oxygen extraction in patients with mild to severe congestive heart failure. Our study determined a maximum oxygen extraction of $78 \%$.

Although as yet unexplained, it is noteworthy that the exercise tolerance did not increase beyond 1.3 mph when the flow rate increased from 100 to 120 $\mathrm{ml} \cdot \mathrm{kg}^{-1} \cdot \mathrm{min}^{-1}$. Similarly, at rest, $\dot{\mathrm{VO}}_{2}$ was higher at $120 \mathrm{ml} \cdot \mathrm{kg}^{-1} \cdot \mathrm{min}^{-1}$ than at $100 \mathrm{ml} \cdot \mathrm{kg}^{-1}$. $\min ^{-1}$, even though both produced equal, low levels of lactate and catecholamines. Clearly, there are details yet to be understood with respect to the distribution and usage of oxygen as perfusion increases with nonpulsatile flow.

Hemoglobin concentration and oxygen delivery. From other studies, one might have expected that anemia could distort the results, as compared with pure hypoxia. ${ }^{38}$ Messmer and associates, ${ }^{39}$ however, showed that hematocrit had to fall below $20 \%$ before lack of cells became a factor.

The animals in this study were somewhat anemic, at an average hemoglobin level of $8.5 \mathrm{gm} / \mathrm{dl}$, as compared with a normal range of 10.3 to 16.0 gm/dl. ${ }^{21}$ The Hemadyne pumps used have previously been reported to produce very little blood damage in similar biventricular studies. ${ }^{8}$ 
In general, across a wide range of hemoglobin concentrations and under an ultimate condition of adequate oxygen delivery, it is believed that $\dot{\mathrm{VO}}_{2}$ is not a function of hemoglobin concentration. As the number of red cells varies, either a changed oxygen extraction or a new cardiac output compensates. In this protocol, the flows were fixed. As shown in Table II, extraction rates varied inversely with hemoglobin content but did not completely compensate and hold the $\mathrm{VO}_{2}$ essentially constant when studied as a function of hemoglobin content. As in the case of increased oxygen delivery by increased flow, the highest hemoglobin levels resulted in higher $\dot{\mathrm{VO}}_{2}$.

Comparison with other nonpulsatile critical flows. To maintain a right atrial pressure below 10 $\mathrm{mm} \mathrm{Hg}$, Yada and associates ${ }^{9}$ determined that a right pump flow of $90 \mathrm{ml} \cdot \mathrm{kg}^{-1} \cdot \min ^{-1}$ was the minimum requirement for normal resting physiology. Several studies ${ }^{9}{ }^{40}$ have shown a $10 \%$ to $20 \%$ difference between right and left flows in chronic nonpulsatile applications. The minimum right flow determined by atrial pressure considerations is therefore consistent with a critical left pump flow of about $100 \mathrm{ml} \cdot \mathrm{kg}^{-1} \cdot \mathrm{min}^{-1}$, established from oxygen transport conditions. These new data are also in agreement with an earlier published comparison of pulsatile and nonpulsatile flow, which suggested that the required flow level was the principal difference between the two modes. ${ }^{41}$

Implications of these results for research and clinical applications. Consistent with previous ani$\mathrm{mal}^{8}$ and human ${ }^{6}$ experiences, data from these five animals show the ability of the mammalian system to adapt to nonpulsatile blood flow. The normal biochemistry results, the basal $\dot{\mathrm{VO}}_{2}$, the maximum oxygen extraction, and the lactate levels show that the overall organ function can be normal with this perfusion regimen, although a slightly higher flow level may be necessary. Even in this study, with nonpulsatile flow of a limited, essentially constant level, the calf could perform a workload of 1.5 METS $\left(\mathrm{VO}_{2}\right.$ max is 1.5 times higher than the basal $\mathrm{VO}_{2}$ ). Clinically, this would permit a very light work load such as shaving, dressing, writing, or slow walking.

We thank Mr. Bryne Wilkerson for his excellent technical assistance.

\section{REFERENCES}

1. Matsumoto T, Wolferth CC Jr, Perlman MH. Effects of pulsatile and non-pulsatile perfusion upon cerebral and conjunctival microcirculation in dogs. Am Surg 1971;37:61-4.
2. Nakayama K, Tamiya T, Yamamoto K, et al. High amplitude pulsatile pump in extracorporeal circulation with particular reference to hemodynamics. Surgery 1963;54:798-809.

3. Hickey PR, Buckley MJ, Philbin DM. Pulsatile and nonpulsatile cardiopulmonary bypass: review of a counterproductive controversy. Ann Thorac Surg 1983;36:720-37.

4. Golding LR, Jacobs G, Groves LK, Gill CC, Nosé Y, Loop FD. Clinical results of mechanical support of the failing left ventricle. J THORAC CARDIOvasc SuRG 1982;83:597-601.

5. Golding LA, Crouch RD, Stewart RW, et al. Postcardiotomy centrifugal mechanical ventricular support. Ann Thorac Surg 1992;54:1059-64.

6. Golding LA, Stewart RW, Sinkewich M, Smith W, Cosgrove DM. Nonpulsatile ventricular assist bridging to transplantation. ASAIO Trans 1988;34:476-9.

7. Yozu R, Golding LA, Jacobs G, Harasaki H, Nosé Y. Experimental results and future prospects for a nonpulsatile cardiac prosthesis. World J Surg 1985;9:116-27.

8. Golding LR, Murakami G, Harasaki $\mathrm{H}$, et al. Chronic nonpulsatile blood flow. Trans Am Soc Artif Intern Organs 1982;28:81-5.

9. Yada I, Golding LR, Harasaki H, et al. Physiopathological studies of nonpulsatile blood flow in chronic models. Trans Am Soc Artif Intern Organs 1983;29:520-5.

10. Schumacker PT, Cain SM. The concept of a critical oxygen delivery. Intensive Care Med 1987;13:223-9.

11. Tominaga R, Smith WA, Massiello A, Harasaki H, Golding LA. Chronic nonpulsatile blood flow. I. Cerebral autoregulation in chronic nonpulsatile biventricular bypass: carotid blood flow response to hypercapnia. J THORAC CARDiovasC SURG 1994;108:907-12.

12. Shepard RB, Kirklin JW. Relation of pulsatile flow to oxygen consumption and other variables during cardiopulmonary bypass. J Thorac Cardiovasc Surg 1969;58:694-702.

13. Shevde K, DeBois WJ. Pro: pulsatile flow is preferable to nonpulsatile flow during cardiopulmonary bypass. J Cardiothorac Anesth 1987;1:165-8.

14. Trinkle JK, Helton NE, Wood RE, Bryant LR. Metabolic comparison of a new pulsatile pump and a roller pump for cardiopulmonary bypass. J THORAC CARDIOVASC SuRG 1969; 58:562-9.

15. Alston RP, Singh M, McLaren AD. Systemic oxygen uptake during hypothermic cardiopulmonary bypass: effects of flow rate, flow character, and arterial $\mathrm{pH}$. J Thorac CARDIOvasC SURG 1989;98:757-68.

16. Singh RK, Barratt-Boyes BG, Harris EA. Does pulsatile flow improve perfusion during hypothermic cardiopulmonary bypass? J Thorac Cardiovasc SuRG 1980;79:827-32.

17. Lunn JK, Liu WS, Stanley TH, Gentry S, Kolff J, Olsen D. Effects of treadmill exercise on cardiovascular and respiratory dynamics before and after artificial heart implantation. Trans Am Soc Artif Intern Organs 1976;22:315-22.

18. Shimomitsu T, Yozu R, Watanabe $T$, et al. Anaerobic threshold for the evaluation of various total artificial heart (TAH) control modes. In: Nosé Y, Kjellstrand C, Ivanovich P, eds. Progress in artificial Organs-1985 Cleveland: ISAO Press, 1986:359.

19. Holliday MA, Potter D, Jarrah A, Bearg S. The relation of metabolic rate to body weight and organ size. Pediatr Res 1967;1:185-95.

20. Hauge A, Nicolaysen G. Pulmonary $\mathrm{O}_{2}$ transfer during 
pulsatile and non-pulsatile perfusion. Acta Physiol Scand 1980;109:325-32.

21. Nelson JH, Ekins DS, Allen SD. Blood characteristics of the Holstein calf relevant in cardiopulmonary studies. J Appl Physiol 1974;37:145-51.

22. Kasnitz P, Druger GL, Yorra F, Simmons DH. Mixed venous oxygen tension and hyperlactatemia. Survival in severe cardiopulmonary disease. JAMA 1976;236:570-4.

23. Simmons DH, Alpas AP, Tashkin DP, Coulson A. Hyperlactatemia due to arterial hypoxemia or reduced cardiac output, or both. J Appl Physiol 1978;45:195-202.

24. Bredle DL, Samsel RW, Schumacker PT, Cain SM. Critical $\mathrm{O}_{2}$ delivery to skeletal muscle at high and low $\mathrm{PO}_{2}$ in endotoxemic dogs. J Appl Physiol 1989;66:2553-8.

25. Cain SM. Oxygen delivery and uptake in dogs during anemic and hypoxic hypoxia. J Appl Physiol 1977;42:228-34.

26. Gutierrez G, Warley AR, Dantzker DR. Oxygen delivery and utilization in hypothermic dogs. J Appl Physiol 1986;60: 751-7.

27. Heusser F, Fahey JT, Lister G. Effect of hemoglobin concentration on critical cardiac output and oxygen transport. Am J Physiol 1989;256:H527-32.

28. Pepe PE, Culver BH. Independently measured oxygen consumption during reduction of oxygen delivery by positive endexpiratory pressure. Am Rev Respir Dis 1985;132:788-92.

29. Shibutani K, Komatsu T, Kubal K, Sanchala V, Kumar V, Bizzarri DV. Critical level of oxygen delivery in anesthetized man. Crit Care Med 1983;11:640-3.

30. Schumacker PT, Long GR, Wood LD. Tissue oxygen extraction during hypovolemia: role of hemoglobin P50. J Appl Physiol 1987;62:1801-7.

31. Wilkens H, Regelson W, Hoffmeister FS. The physiologic importance of pulsatile blood flow. N Engl J Med 1962;267: $443-6$.
32. Ogata T, Ida Y, Nonoyama A, Takeda J, Sasaki H. A comparative study of the effectiveness of pulsatile and nonpulsatile flow in extracorporeal circulation. Arch Jpn Clin 1960;29:59-66.

33. Krogh $\mathrm{A}$. The number and distribution of capillaries in muscle with calculations of the pressure head necessary for supplying the tissue. J Physiol (Lond) 1919;52:409-15.

34. Roca J, Hogan MC, Story D, et al. Evidence for tissue diffusion limitation of $\mathrm{VO}_{2}$ max in normal humans. J Appl Physiol 1989;67:291-9.

35. Weber KT, Kinasewitz GT, Janicki JS, Fishman AP. Oxygen utilization and ventilation during exercise in patients with chronic cardiac failure. Circulation 1982;65:1213-23.

36. Weber KT, Janicki JS. Lactate production during maximal and submaximal exercise in patients with chronic heart failure. J Am Coll Cardiol 1985;6:717-24.

37. Wilson JR, Martin JL, Schwartz D, Ferraro N. Exercise intolerance in patients with chronic heart failure: role of impaired nutritive flow to skeletal muscle. Circulation 1984; 69:1079-87.

38. Moss M, Kurzner S, Razlog Y, Lister G. Hypoxanthine and lactate concentrations in lambs during hypoxic and stagnant hypoxia. Am J Physiol 1988;255:H53-9.

39. Messmer K, Sunder-Plassman L, Jesch F, Gornandt L, Sinagowitz E, Kessler M. Oxygen supply to the tissues during limited normovolemic hemodilution. Res Exp Med (Berl) 1973;159:152-66.

40. Takatani S, Koike S, Yada I, et al. Left and right pump flow differences in nonpulsatile biventricular bypass and total artificial heart animal. Jpn Artif Organs 1983;12:16-9.

41. Valdes F, Takatani S, Jacobs GB, et al. Comparison of hemodynamic changes in a chronic nonpulsatile biventricular bypass and total artificial heart (TAH). Trans Am Soc Artif Intern Organs 1980;26:455-60. 